University of New Hampshire

University of New Hampshire Scholars' Repository

Space Science Center

Institute for the Study of Earth, Oceans, and

Space (EOS)

7-1997

\title{
Activation in the COMPTEL double-scattering gamma-ray telescope
}

\author{
D Morris \\ University of New Hampshire - Main Campus \\ Mark L. McConnell \\ University of New Hampshire - Main Campus, mark.mcconnell@unh.edu \\ James M. Ryan \\ University of New Hampshire, James.Ryan@unh.edu \\ R Diehl \\ Max-Planck-Institut für extraterrestriche Physik \\ U Oberlack \\ Max-Planck-Institut für extraterrestrische Physik
}

See next page for additional authors

Follow this and additional works at: https://scholars.unh.edu/ssc

Part of the Astrophysics and Astronomy Commons

\section{Recommended Citation \\ Morris, D.J.; McConnell, M.L.; Ryan, J.M.; Diehl, R.; Oberlack, U.G.; Schonfelder, V.; Varendorff, M.; Weidenspointner, G.; De Boer, H.; Bennett, K., "Activation in the COMPTEL double-scattering gamma-ray telescope," High Energy Radiation Background in Space, 1997 Conference on the , vol., no., pp.26,29, 22-23 Jul 1997 doi: 10.1109/CHERBS.1997.660241}

This Conference Proceeding is brought to you for free and open access by the Institute for the Study of Earth, Oceans, and Space (EOS) at University of New Hampshire Scholars' Repository. It has been accepted for inclusion in Space Science Center by an authorized administrator of University of New Hampshire Scholars' Repository. For more information, please contact Scholarly.Communication@unh.edu. 


\section{Authors}

D Morris, Mark L. McConnell, James M. Ryan, R Diehl, U Oberlack, V Schonfelder, M Varendorff, G Weidenspointer, $\mathrm{H}$ deBoer, and $\mathrm{K}$ Bennett 


\title{
Activation in the COMPTEL Double-Scattering Gamma-Ray Telescope
}

\author{
D.J. Morris, M.L. McConnell, J.M. Ryan \\ Space Science Center, University of New Hampshire, Durham NH 03824-3525 USA
}

R. Diehl, U.G. Oberlack, V. Schönfelder, M. Varendorff, G. Weidenspointner
Max-Planck-Institut für extraterrestrische Physik, Postfach 1603, D-85740 Garching, Germany

H. de Boer

SRON-Utrecht, Sorbonnelaan 2, NL-3584 CA Utrecht, The Netherlands

K. Bennett

Astrophysics Division, Space Science Department of ESA/ESTEC, NL-2200 AG Noordwijk, The Netherlands

\begin{abstract}
The COMPTEL gamma-ray telescope has been operating in low Earth orbit for six years, since the launch of the Compton Gamma-Ray Observatory in April 1991. Comparisons of data for different orbits and epochs show evidence of activation on time scales from minutes $\left({ }^{27} \mathrm{Mg}, \tau_{1 / 2}=9.5 \mathrm{~min}\right)$ to years $\left({ }^{22} \mathrm{Na}, \tau_{1 / 2}=2.58 \mathrm{yr}\right.$ ). The activation is correlated with both the orbital altitude and solar cosmic-ray modulation. Because it requires coincident measurements in two different detectors, COMPTEL is most susceptible to instrumental background events in which two or more photons are produced simultaneously.
\end{abstract}

\section{INTRODUCTION}

COMPTEL is a double-scattering telescope designed for gamma-ray astronomy in the energy range $0.75-30 \mathrm{MeV}$ [1]. It has two sets of detector modules arrayed in two planes separated by about $1.5 \mathrm{~m}$ (Fig. 1). The upper (D1) detector consists of seven cylindrical modules filled with NE213A liquid scintillator, each with radius $13.8 \mathrm{~cm}$ and depth $8 \mathrm{~cm}$ and viewed by eight photomultipliers around the side. The lower (D2) detector consists of 14 cylindrical $\mathrm{NaI}$ modules, each with radius $14 \mathrm{~cm}$ and depth $7.5 \mathrm{~cm}$ and viewed from below by seven photomultipliers. Each detector is surrounded by plastic charged-particle shields. There are also two calibration (CAL) units, each composed of a ${ }^{60} \mathrm{Co}$-doped plastic scintillator wafer viewed by two half-inch photomultiplier tubes, located on opposite sides of the telescope halfway between D1 and D2. These provide tagged photons for in-flight energy calibration.

An incident photon is detected when it scatters in the D1 detector followed by an interaction of the scattered photon in the D2 detector. Ideally, the scattered photon is absorbed in a D2 module. The total energy measured in the two detectors is then the energy of the incident photon, and the ratio of the energies in the two detectors, together with the interaction locations in the detectors, allow the direction of the incident photon to be localized to a circle on the sky. The time between the two interactions (TOF) and the pulse shape in the D1 detector are used to discriminate against events which are not

This work is supported by NASA under contract NASS-26645, by the German government through DARA grant 50 QV 90968 and by the Netherlands Organization for Scientific Research (NWO). caused by single photons from within the normal field of view, extending about $40^{\circ}$ from the telescope axis.

COMPTEL is one of four experiments aboard the Compton Gamma-Ray Observatory (CGRO), which was launched on April 5, 1991 into a circular orbit with inclination $28^{\circ}$. Until recently, the altitude of the orbit was maintained between 330 and $450 \mathrm{~km}$. During its first 18 months of operation COMPTEL completed a survey of the gamma-ray sky. This was followed by observations of sources or regions of particular interest. Observing periods typically last one to two weeks, during which the orientation of the spacecraft is maintained toward a given point on the sky. In April 1997 the spacecraft was boosted to an altitude of $515 \mathrm{~km}$ which should allow it to continue operating through the coming solar maximum without further orbital adjustments.

Despite the measures to reject events not produced by ce-

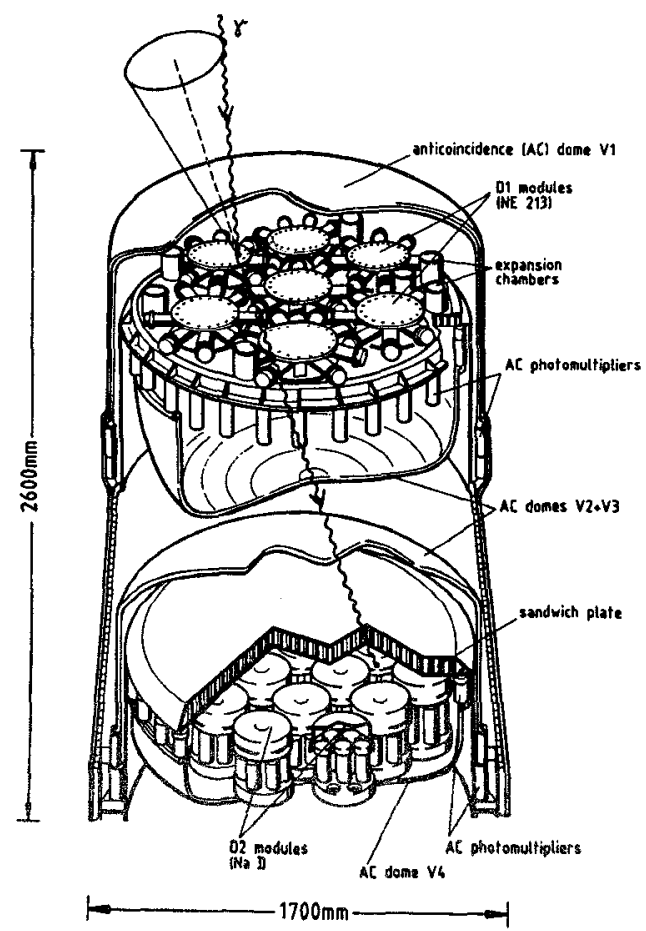

Fig. 1: Schematic diagram of COMPTEL 


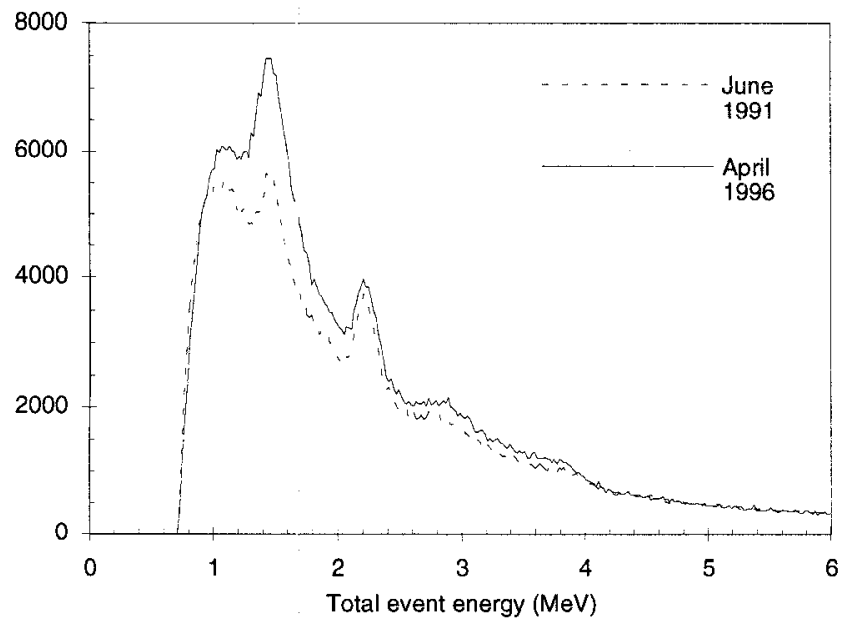

Fig. 2: Count spectra in total energy for two 14-day observations in June 1991 and April 1996.

lestial photons, the COMPTEL data are dominated by instrumental background. Fig. 2 shows two typical count spectra of acceptable double-scatter events, from June 1991 and April 1996. The bulk of the events form a smooth distribution with an exponential decline following the sharp rise near the energy threshold. These events are mostly prompt background, which is the subject of another talk at this conference [2]. There are two very prominent peaks on top of the smooth continuum. At $2.2 \mathrm{MeV}$ is the deuterium-formation line from the combination of thermal neutrons with hydrogen in the NE213A liquid scintillator [3]. There is a more complex feature near $1.5 \mathrm{MeV}$. Part of it is due to the decay of primordial ${ }^{40} \mathrm{~K}$ in the Dl photomultiplier tubes, but it is too wide and too large to be explained by this single decay line. Its growth in both width and amplitude since launch, which can be clearly seen in Fig. 2, indicates that activation is responsible for a large fraction of the background in this energy range. In-flight activation also produces a number of smaller spectral features at energies up to $4 \mathrm{MeV}$.

\section{CHARA.CTERISTICS OF ACTIVATION}

Most activation events share certain characteristics which allow them to evade the background discrimination measures. The isotopes involved produce two or more photons simultaneously (in an interval short compared to the D1-D2 time-offlight). These may be two decay photons or a single decay photon plus a bremsstrahlung photon (produced by a $\beta$-particle) or annihilation photon. The photon energies must be sufficient to deposit the threshold energies of $70 \mathrm{keV}$ in D1 and $650 \mathrm{keV}$ in D2. In order for the event to have an acceptable TOF, the activation must occur in or near the D1-detector modules. The materials in which activation is of most concern are thus the D1 housings and support structure (Al), the D1 photomultipliers and the quartz windows in the housings (mostly $\mathrm{Si}$ and $\mathrm{O}$, but also $\mathrm{Cu}$ and other elements in the photomultipliers) and the liquid scintillator itself (C). Table 1 lists those isotopes for which evidence has been found. These and other isotopes may also produce single-photon events,
TABLE 1

IMPORTANT ACTIVATION SPECIES

\begin{tabular}{|c|c|c|c|}
\hline Isotope & $\tau_{1 / 2}$ & $\begin{array}{c}\text { Decay modes and } \\
\text { photon energies }(\mathrm{MeV})\end{array}$ & $\begin{array}{c}\text { Production } \\
\text { channels }\end{array}$ \\
\hline${ }^{22} \mathrm{Na}$ & $3.76 \mathrm{yr}$ & $\begin{array}{r}\beta^{+}(89 \%) ; \mathrm{e}^{-} \text {capture }(11 \%) \mathrm{E}_{\gamma} \\
=0.511,1.275\end{array}$ & $\begin{array}{c}{ }^{27} \mathrm{Al}(\mathrm{p}, 3 \mathrm{p} 3 \mathrm{n}) \\
+ \text { others }\end{array}$ \\
\hline${ }^{24} \mathrm{Na}$ & $15.0 \mathrm{hr}$ & $\beta, \mathrm{E}_{\gamma}=1.37,2.753$ & $\begin{array}{c}{ }^{27} \mathrm{Al}(\mathrm{n}, \boldsymbol{\alpha}) \\
\mathrm{Al}(\mathrm{p}, 3 \mathrm{pn})\end{array}$ \\
\hline${ }^{27} \mathrm{Mg}$ & $9.5 \mathrm{~min}$ & $\beta, \mathrm{E}_{\gamma}=0.844(72 \%)$, & ${ }^{27} \mathrm{Al}(\mathrm{n}, \mathrm{p})$ \\
\hline${ }^{28} \mathrm{Al}$ & $2.3 \mathrm{hr}$ & $\beta, 014(28 \%)$ & $\mathrm{E}_{\gamma}=1.78$ \\
\hline
\end{tabular}

though most are rejected as coming from outside the telescope's field of view.

Firm identification of activation features requires a detailed examination of the data. Features can be accentuated by selecting and comparing subsets of the data for particular periods. Activation is expected to be most intense when the spacecraft passes through the South-Atlantic Anomaly (SAA). The typical daily fluence of trapped protons in the SAA with energies $>100 \mathrm{MeV}$ on a $450 \mathrm{~km}$ orbit was expected to be near $1.5 \times 10^{6} \mathrm{~cm}^{-2}$ [4], about 100 times the daily fluence of cosmic-ray protons. All instrument components are switched off during SAA passages except the CAL units, which provide a rough measure of the charged particle flux through the telescope.

Short-lived isotopes such as ${ }^{27} \mathrm{Mg}$ are expected to be most evident in the periods immediately following SAA passages, though activation continues at a lower level elsewhere. Longlived isotopes such as ${ }^{22} \mathrm{Na}$ build up over a period comparable to their halflife, at a rate which depends on both the spacecraft altitude and solar modulation of the charged-particle fluxes. Comparisons of data from different epochs may reveal longlived activation.

Activation features from two-photon events are best studied using quantities other than the total event energy. Individual activation lines can be identified by their photopeaks in D2-module energy (E2) spectra. The corresponding energy deposits in D1 (E1), most often from a single Compton scattering, will span a range from the $70-\mathrm{keV}$ threshold to the Compton edge, with a multiple-scattering tail extending to the full photon energy. The TOF distribution expected for two-photon activation events differs from that for single-photon events, being wider and peaking at a slightly lower TOF value. Monte Carlo simulations of activation events have proved very helpful for associating features in the data with particular isotopes.

\section{EVIDENCE FOR INDIVIDUAL ISOTOPES}

\section{A. ${ }^{24} \mathrm{Na}$}

Two features in the total-energy spectrum associated with ${ }^{24} \mathrm{Na}$ activation were recognized early in the mission. Both 


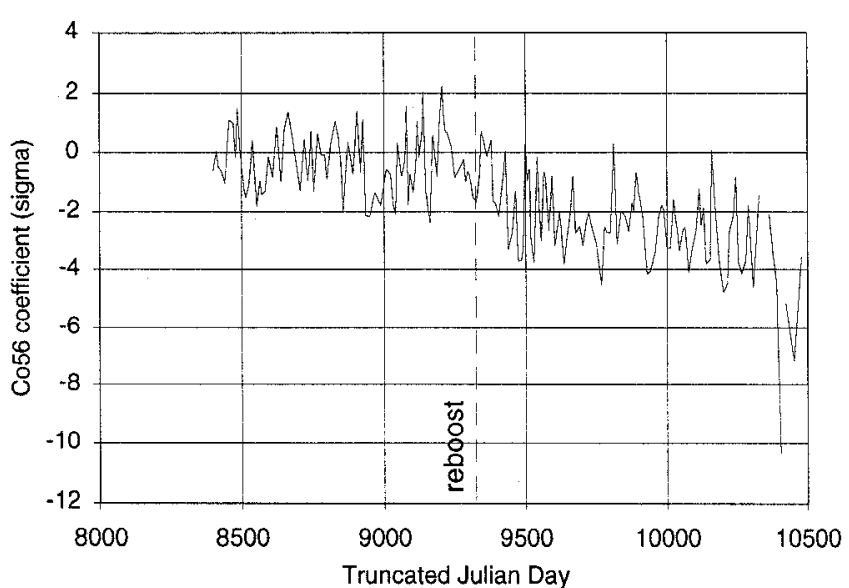

Fig. 3: Coefficient of the ${ }^{56}$ Co template in sigma, in the spectral fitting of data for individual observation periods. The time of the spacecraft reboost is indicated.

can be discerned in Fig. 2, near $2.8 \mathrm{MeV}$ and $3.9 \mathrm{MeV}$. The $2.8-\mathrm{MeV}$ feature comes from the absorption of the $2.75-\mathrm{MeV}$ photon in D2, accompanied by scattering of the $1.37-\mathrm{MeV}$ photon in D1 with an energy deposit near the D1 threshold. The complementary process, with absorption of the 1.37$\mathrm{MeV}$ photon in $\mathrm{D} 2$, contributes to the $1.5-\mathrm{MeV}$ feature. The 3.9-MeV feature comes from scattering of either photon in D1, with an energy deposit near the Compton edge, accompanied by absorption of the other in D2. Confirmation that these features are due to ${ }^{24} \mathrm{Na}$ was obtained by analyzing the event distribution in E1-E2 space. Also, TOF distributions produced using energy selections which favor ${ }^{24} \mathrm{Na}$ events were found to have the characteristics expected of two-photon activation.

\section{B. ${ }^{27} \mathrm{Mg}$}

The isotope ${ }^{27} \mathrm{Mg}$ is produced by secondary neutrons from the atmosphere or spacecraft, with a cross section comparable to the ${ }^{27} \mathrm{Al}(\mathrm{n}, \alpha)^{24} \mathrm{Na}$ reaction. But it was not recognized so soon as ${ }^{24} \mathrm{Na}$, which is also produced directly by energetic protons. The first indications were found during a search for cosmic line emission from the two principal lines of ${ }^{56} \mathrm{Co}$ at 847 and $1238 \mathrm{keV}$. The most obvious ${ }^{27} \mathrm{Mg}$ events should be produced by the absorption of the decay photon, with energy $844 \mathrm{keV}$ or $1014 \mathrm{keV}$, in D2 accompanied by the scattering of a bremsstrahlung photon in D1. These events will extend upward in total energy from $894 \mathrm{keV}$ and $1064 \mathrm{keV}$, filling the gap between the ${ }^{56} \mathrm{Co}$ lines.

The most sensitive technique for detecting ${ }^{56} \mathrm{Co}$ was found to be the fitting of total-energy spectra with an analytic function, to approximate the background, plus a ${ }^{56} \mathrm{Co}$-emission template [5]. This spectral-fitting technique has proven useful in background studies as well. It was used to quantify the growth of the $1.5-\mathrm{MeV}$ feature. Also, deviations from the background model which are persistent but variable in amplitude are indications of possible activation.

In the vicinity of the ${ }^{56} \mathrm{Co}$ lines, the background model was generally satisfactory for observations up to late 1993, when the spacecraft was boosted in altitude from $330 \mathrm{~km}$ to

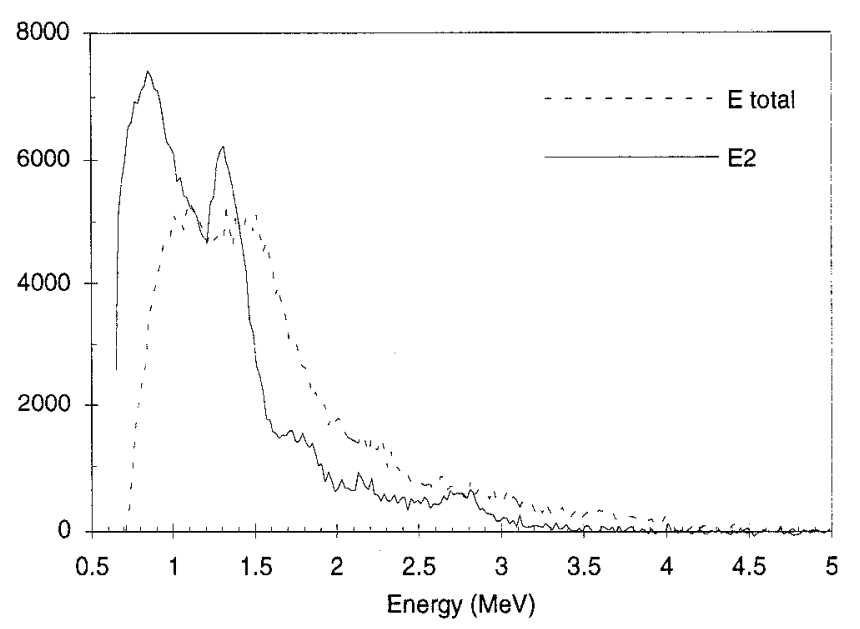

Fig. 4: Residual counts in the first 30 min following SAA passages for 148 days of data from April to October 1996. Spectra are shown for both the total energy and the D2 energy.

$450 \mathrm{~km}$. In subsequent observations, the fitted coefficient for the ${ }^{56} \mathrm{Co}$ template usually has a significant negative value, as shown in figure 3 , though with considerable variability. Fits of those spectra without the emission template showed positive deviations between 850 and $1150 \mathrm{keV}$, where the ${ }^{27} \mathrm{Mg}$ activation features were expected. The variable nature of the feature also suggested a short-lived isotope, such as ${ }^{27} \mathrm{Mg}$.

The primary effect on the spacecraft radiation environment expected from the reboost was an increase in the energetic proton fluence encountered during SAA passages, especially at low energies. While the spacecraft was also at $450 \mathrm{~km}$ immediately following launch, that was in a period of intense solar activity, which reduced both the cosmic-ray and trapped-particle fluences.

If most ${ }^{27} \mathrm{Mg}$ activation occurs in the SAA then, given its 9.5-min halflife, most ${ }^{27} \mathrm{Mg}$ events should be found in short periods following the SAA passages. To test this hypothesis, spectra were accumulated for a number of recent observations, omitting $30 \mathrm{~min}$ following each SAA passage and compared to spectra for the full observations. Fig. 4 shows the excess counts in both E2 and total energy following SAA passages, summed over 148 days of data from April to October 1996. The data were normalized to the counts at total energy 10-30 MeV, a range with much prompt background, but expected to be free of activation. The excess counts are near zero through the range 5-10 $\mathrm{MeV}$, confirming that the normalization is correct. But below $4 \mathrm{MeV}$ total energy, an excess of activation events is apparent. This excess accounts for about $30 \%$ of the events up to $1.7 \mathrm{MeV}$ in the $30 \mathrm{~min}$ following SAA passages. The E2 spectrum is more informative as to the sources of the activation. It has a peak near the energy of the more common ${ }^{27} \mathrm{Mg}$ photon $(844 \mathrm{keV}$ ) with a high-energy shoulder extending past the energy of the other $(1014 \mathrm{keV})$. But there are features consistent with the $\gamma$ emission of ${ }^{24} \mathrm{Na}$ and ${ }^{28} \mathrm{Al}$ as well.

Using the spectral-fitting method, $36 \%$ of the events in the ${ }^{27} \mathrm{Mg}$ feature are estimated to occur in the $30 \mathrm{~min}$ after SAA passages. While this is almost twice the fraction of high-en- 


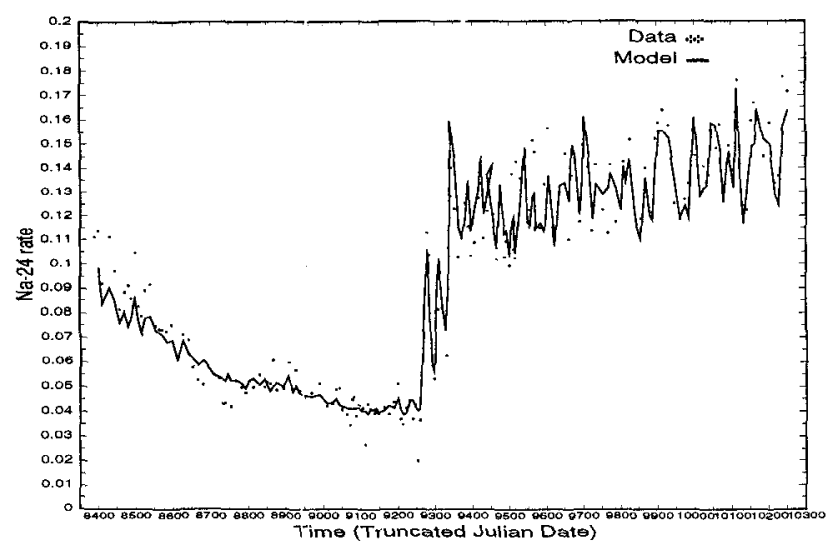

Fig. 5: Model of the ${ }^{24} \mathrm{Na}$ activation with the measured ${ }^{24} \mathrm{Na}$ event rates [8].

ergy events in those periods (19\%), it is much less than the $90 \%$ expected if all ${ }^{27} \mathrm{Mg}$ activation took place in the SAA. This may imply that, while the intensity of ${ }^{27} \mathrm{Mg}$ activation is greatest in the SAA, most occurs during the longer exposure outside the SAA. This is not unreasonable for species produced exclusively by neutrons. But in that case, it is not clear why the ${ }^{27} \mathrm{Mg}$ activation increased so much following the reboost. There may well be other isotopes which contribute to activation near $1 \mathrm{MeV}$ which have not yet been identified.

\section{C. ${ }^{22} \mathrm{Na}$}

The ${ }^{22} \mathrm{Na}$ activation was discovered during processing of an all-sky database for the cosmic ${ }^{26} \mathrm{Al}$ emission line at 1.809 $\mathrm{MeV}[6,7]$. The first indication was an enhancement of the events in the energy windows used for background, 1.6-1.7 $\mathrm{MeV}$ and 1.9-2.0 MeV, relative to the $1.7-1.9 \mathrm{MeV}$ source window. Like the appearance of the ${ }^{27} \mathrm{Mg}$ feature and the growth of the $1.5-\mathrm{MeV}$ feature, this began following the 1993 reboost. It seemed likely that activation events in the wing of the $1.5-\mathrm{MeV}$ feature were adding to the $1.6-1.7 \mathrm{MeV}$ background.

Most ${ }^{22} \mathrm{Na}$ events result from the absorption of the 1275 $\mathrm{keV}$ photon in D2 together with scattering of one $511-\mathrm{keV}$ annihilation photon in D1. The total energy for such an event, with a Compton-edge energy deposit in D1, falls in the 1.6-1.7 MeV window. Confirmation of ${ }^{22} \mathrm{Na}$ activation was obtained by examining event distributions in E1-E2 space for observations preceding and following the reboost. In E2 spectra for events with $E 1=300-370 \mathrm{keV}$, around the Compton edge for the $511-\mathrm{keV}$ photon $(340 \mathrm{keV})$, a complex feature was found at $1200-1500 \mathrm{keV}$. This feature was fit with a combination of two Gaussians, one fixed at $1369-\mathrm{keV}$, the energy of one ${ }^{24} \mathrm{Na}$ photon. The position derived for the other was $1272.8 \pm 2.6 \mathrm{keV}$, consistent with the $1274.6-\mathrm{keV}$ energy of the ${ }^{22} \mathrm{Na}$ decay photon. There is probably a third component in this feature which has not yet been identified.

\section{D. ${ }^{28} A l$}

The evidence for ${ }^{28} \mathrm{Al}$ activation is a Gaussian feature seen near $1800 \mathrm{keV}$ in E'2 spectra, like that in Fig. 4. It would be surprising if this isotope were not seen, but more detailed study is needed to confirm that the $1800-\mathrm{keV} \mathrm{E} 2$ feature is indeed due to ${ }^{28} \mathrm{Al}$. Though the halflife of ${ }^{28} \mathrm{Al}, 2.3 \mathrm{hr}$, is much longer than that of ${ }^{27} \mathrm{Mg}$, the accentuation of the $1800-\mathrm{keV}$ feature in the 30 -min periods following SAA passages indicates that ${ }^{28} \mathrm{Al}$ activation is associated with the SAA. The SAA passages occur on six or seven successive orbits per day, with an orbital period of about $1.5 \mathrm{hr}$. The ${ }^{28} \mathrm{Al}$ feature might be more clearly seen in the excess counts for 10-12 hr periods beginning with the first SAA passage in each group.

\section{ACTIVATION RATES}

Rates for both ${ }^{22} \mathrm{Na}$ and ${ }^{24} \mathrm{Na}$ events have been derived for each CGRO observing period. The temporal variation of these event rates can be predicted by using the CAL-unit rate as a proxy for the activation rate. Both event rates follow the predictions [8]. Because of its short halflife, the ${ }^{24} \mathrm{Na}$ event rates, shown in Fig. 5, closely tracks the CAL-unit rates. The increase in activation following the 1993 reboost is obvious. The event rate for long-lived ${ }^{22} \mathrm{Na}$, in contrast, has risen smoothly and monotonically. The rate of increase fell to near zero just before the reboost, but the increase then resumed at a high level which has been maintained since. A daily proton fluence through the telescope of $2.3 \times 10^{5}$ protons $(\mathrm{E}>100$ $\mathrm{MeV}$ ) $\mathrm{cm}^{-2}$ is estimated from the ${ }^{22} \mathrm{Na}$ activation rate for an orbital altitude of $440 \mathrm{~km}$ in April 1991.

\section{CONCLUSIONS}

Though the requirement for coincident events in two detectors, along with other measures, reduces instrumental background in COMPTEL, activation remains an important concern at event energies below $4 \mathrm{MeV}$. The activation of ${ }^{22} \mathrm{Na}$ and ${ }^{24} \mathrm{Na}$ has been studied in detail, and occurs at a level consistent with expectations. There is good evidence as well for activation of ${ }^{27} \mathrm{Mg}$ and ${ }^{28} \mathrm{Al}$. Further study is needed to determine the activation rates for the latter two isotopes and identify others that may be present. Better understanding of the activation will allow optimization of COMPTEL astronomical data analysis and improved design for future instruments.

\section{REFERENCES}

[1] V. Schönfelder et al., "Instrument description and performance of the imaging gamma-ray telescope COMPTEL aboard NASA's Compton gamma-ray observatory", Astrophys. J. Suppl., 86, 657 (1993).

[2] J.M. Ryan et al., "The prompt cosmic-ray induced background in the orbiting Compton telescope COMPTEL", in these proceedings.

[3] G. Weidenspointner et al., "The local neutron flux at low earth orbiting altitudes", submitted to Advances in Space Research.

[4] C.S. Dyer, P.R. Truscott, N.D.A. Hammond and C. Comber, "Radioactivity induced in gamma-ray spectrometers", in High-Energy Radiation Background in Space, eds. A.C. Rester and J.I. Trombka, New York: American Institute of Physics, 1989, pp. 278-288.

[5] D.J. Morris et al., "Evidence for ${ }^{56} \mathrm{Co}$ line emission from the type Ia supernova 1991T using COMPTEL", in Seventeenth Texas Symposium on Relativistic Astrophysics and Cosmology, eds. H. Böhringer et al., New York: N.Y. Academy of Sciences, 1995, pp. 397-400.

[6] U. Oberlack, thesis, in preparation.

[7] U. Oberlack et al., "A new background model for COMPTEL 1.8-MeV data", to be published in the Proceedings of the 4th Compton Symposium, Williamsburg VA, 1997.

[8] M. Varendorff et al., "A time-dependent model for the activation of COMPTEL", to be published in the Proceedings of the 4th Compton Symposium, Williamsburg VA, 1997. 